\title{
Subnucleon fluctuations in vector meson photoproduction: a window to saturation
}

\author{
J. Cepila ${ }^{a}$, J. G. Contreras ${ }^{* a}$, M. Krelina ${ }^{a b}$ and J. D. Tapia Takaki ${ }^{c}$ \\ ${ }^{a}$ Faculty of Nuclear Sciences and Physical Engineering, \\ Czech Technical University in Prague, Prague, Czech Republic \\ ${ }^{b}$ Departamento de Física, Universidad Técnica Federico Santa María; \\ Centro Científico-Tecnológico de Valparaíso-CCTVal, \\ Casilla 110-V, Valparaíso, Chile \\ ${ }^{c}$ Department of Physics and Astronomy, The University of Kansas, Lawrence, KS, USA \\ E-mail: jgcn@mail.cern.ch
}

\begin{abstract}
We present a model of coherent and incoherent photoproduction of vector mesons off proton and off nuclear targets. The computations are performed within the colour-dipole formalism. The targets are described by a profile, in the impact parameter space, including subnucleon degrees of freedom, so-called hot spots. Their positions change event by event. The key aspect of our model is that the number of hot spots grows with energy.

We show that the measurement of dissociative production off protons at different energies offers new signatures of saturation. These measurements can be performed with current HERA and LHC data. The results presented here are based on our previous studies published in [1],[2], and [3].
\end{abstract}

XXVI International Workshop on Deep-Inelastic Scattering and Related Subjects (DIS2018) 16-20 April 2018

Kobe, Japan

${ }^{*}$ Speaker. 

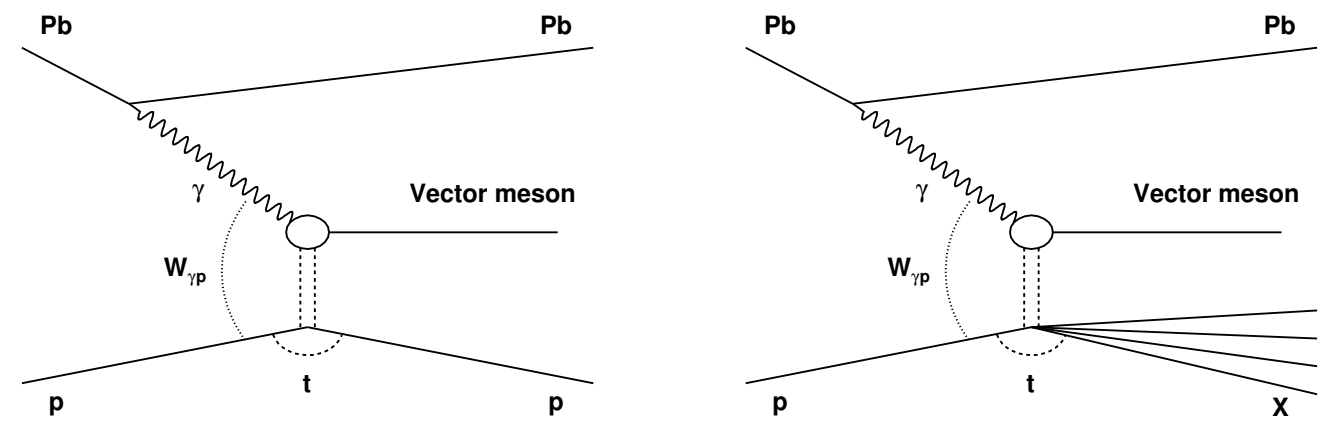

Figure 1: Schematic representation for exclusive (left) and dissociative (right) vector meson photoproduction. The processes are depicted as seen at the LHC, where the source of photons is a lead ion. At HERA, the source of photons was either an electron or a positron.

\section{Introduction}

The high-energy, equivalently small- $x$, limit of hadron structure is one of the most interesting subjects of perturbative QCD today, because saturation is expected to be found in this domain. In this context, saturation means a dynamical equilibrium between the production and the recombination of gluons carrying a very small fraction of the total momentum of their parent hadron.

Hadrons are made of relativistic quantum fields and as such their structures fluctuate. Similar experiments will probe different configurations each time. In these proceedings we present predictions for a process that is sensitive to spatial geometrical fluctuations in the impact parameter plane, namely the diffractive production of a vector meson in photon-hadron interactions.

Specifically, we work within the colour-dipole model to compute exclusive and dissociative production of $\rho^{0}, \mathrm{~J} / \psi$ and $\Upsilon(1 S)$ vector mesons off protons. A schematic representation of these processes is shown in Fig. 1, where $W_{\gamma p}$ denotes the centre-of-mass energy of the photon-proton interaction and $t$ the square of the four-momentum transferred at the proton vertex. In addition, we study the corresponding processes - called coherent and incoherent, respectively — for photonuclear production of $\rho^{0}$. Our model includes a prescription for saturation as proposed by GolecBiernat and Wuesthoff [4] and a so-called hot-spot structure in the impact parameter plane, where the hot spots represent regions of high gluon density.

These proceedings contain results from our previous studies published in [1],[2], and [3]. The rest of this contribution is organised as follows. Next section introduces briefly the model, Sec. 3 and Sec. 4 contain our results for proton and for nuclear targets, respectively, and in Sec.5 we present our conclusions and an outlook.

\section{Brief overview of the model}

The cross sections for the photoproduction of a vector meson VM off a target $\mathrm{T}$ for a photon of virtuality $Q^{2}$, and transverse $(T)$ or longitudinal $(L)$ polarisation are

$$
\left.\frac{d \sigma(\gamma \mathrm{T} \rightarrow \mathrm{VMT})}{d t}\right|_{T, L}=\frac{\left(R_{g}^{T, L}\right)^{2}}{16 \pi}\left|\left\langle A\left(x, Q^{2}, \vec{\Delta}\right)_{T, L}\right\rangle\right|^{2}
$$


for the exclusive or coherent processes, and

$$
\left.\frac{d \sigma(\gamma \mathrm{T} \rightarrow \mathrm{VM} Y)}{d t}\right|_{T, L}=\frac{\left(R_{g}^{T, L}\right)^{2}}{16 \pi}\left(\left\langle\left|A\left(x, Q^{2}, \vec{\Delta}\right)_{T, L}\right|^{2}\right\rangle-\left|\left\langle A\left(x, Q^{2}, \vec{\Delta}\right)_{T, L}\right\rangle\right|^{2}\right),
$$

for dissociative or incoherent production, where $Y$ represents the dissociative state. $R_{g}^{T, L}$ is the skewedness correction [5], $\Delta^{2} \equiv-t$ and $x=M_{\mathrm{VM}}^{2} / W_{\gamma \mathrm{T}}^{2}$ with $M_{\mathrm{VM}}$ the mass of the vector meson. The total cross section at a given $t$ is the sum of the $T$ and $L$ contributions. The integral over $t$ yields the cross section at a given energy. The angular brackets represent an average over different geometrical configurations of the hot spots in the plane transverse to the interaction.

The amplitude takes the standard form for the colour-dipole model (for details see e.g. [1]). Here we want to concentrate on the cross section for the interaction, at an impact parameter $\vec{b}$, of a dipole of transverse size $\vec{r}$ with the target. For proton targets we use

$$
\frac{d \sigma_{\mathrm{dp}}}{d \vec{b}}=2 \sigma_{0} N(x, r) T(\vec{b})
$$

while for nuclei

$$
\frac{d \sigma_{\mathrm{dA}}}{d \vec{b}}=2\left[1-\exp \left(-\frac{1}{2} \sigma_{0} N(x, r) T_{\mathrm{A}}(\vec{b})\right)\right] .
$$

In both cases, the fluctuations are implemented in the profile functions $T(\vec{b})$ and $T_{\mathrm{A}}(\vec{b})$. For the proton we use

$$
T(\vec{b})=\frac{1}{N_{h s}} \sum_{i=1}^{N_{h s}} T_{h s}\left(\vec{b}-\vec{b}_{i}\right)
$$

with

$$
T_{h s}\left(\vec{b}-\vec{b}_{i}\right)=\frac{1}{2 \pi B_{h s}} e^{-\frac{\left(\vec{b}-\vec{b}_{i}\right)^{2}}{2 B_{h s}}},
$$

where the position of a hot spot $(h s)$ is a random point extracted form a 2D-Gaussian distribution centred at zero and of width $B_{p}$ related to the size of the proton. For the nuclear case we sample the position of nucleons using random points from a Wood-Saxon distribution where each nucleon has a hot-spot structure as just described.

The key ingredient of our approach is the fact that the number of hot spots inside a nucleon increases with decreasing values of $x$. This is shown schematically in Fig 2. The size of the hot spot does not change as shown in the left part of the figure.

\section{Photoproduction off protons}

Figure 3 shows the results of our model for the exclusive and dissociative photoproduction of $\rho^{0}, \mathrm{~J} / \psi$ and $\Upsilon(1 S)$ vector mesons off protons as a function of $W_{\gamma_{\mathrm{p}}}$ as computed in our model (solid lines) compared to data from HERA $[6,7,8,9,10]$ and the LHC $[11,12,13]$.

The cross sections for the exclusive photoproduction of vector mesons are satisfactorily described over a large energy range and an even larger range in cross sections. Except for the slope of the $t$ distribution in the case of $\rho$ production, all parameters of the model are the same for the three vector mesons. For the $\rho^{0}$ we had to increase the slope from $4.7 \mathrm{GeV}^{-2}$ to $8 \mathrm{GeV}^{-2}$ to obtain a good description of the $t$ distribution at one value of $W_{\gamma \mathrm{p}}$. (See [3] for details.) 

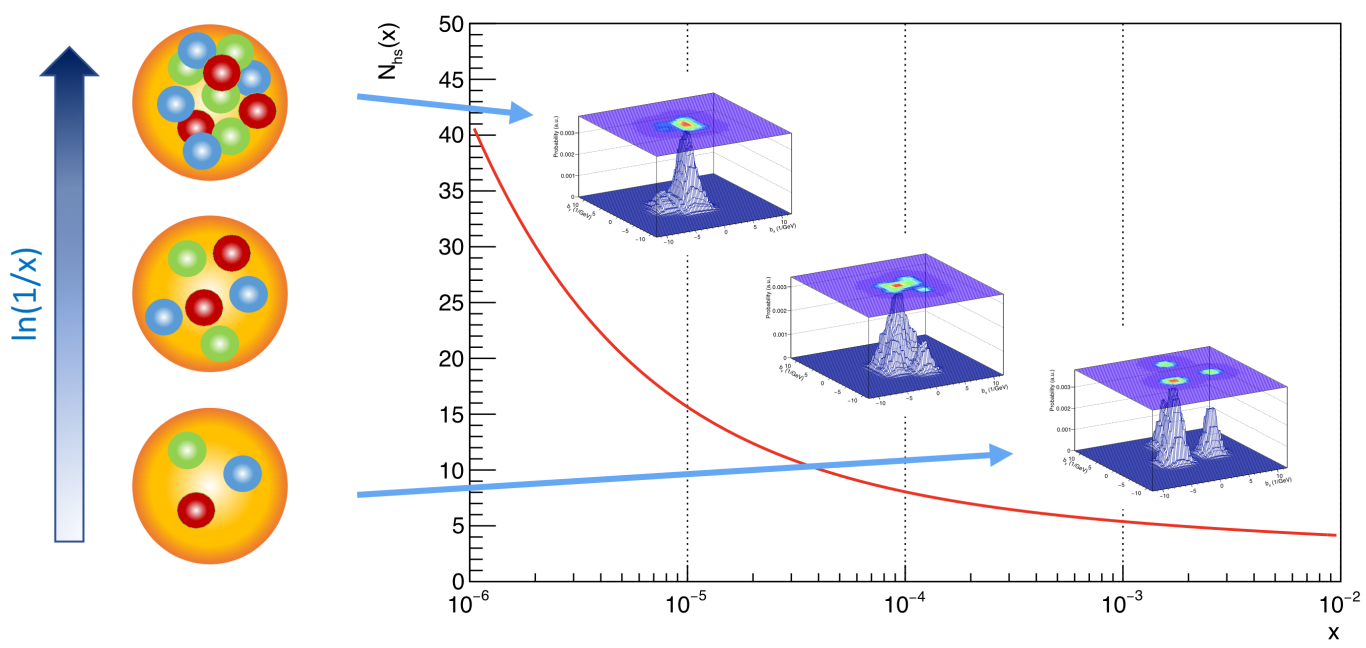

Figure 2: Schematic representation of the growth in the number of hot spots with decreasing values of $x$. In the left, there is a cartoon of particular hot-spot configurations inside the hadron at different values of $x$. In the right part of the figure, the curve shows the actual dependence used for calculations and three examples of $T(\vec{b})$ distributions.

Our main result is shown in the right panel of Fig. 3. The dissociative cross section shows (i) a marked decreased at large energies and (ii) the place where this decrease start, which depends on the mass of the vector meson: it is at a smaller $W_{\gamma p}$ for the $\rho^{0}$, at a larger $W_{\gamma p}$ for the $\mathrm{J} / \psi$, and at even larger energies for the $\Upsilon(1 S)$. The most interesting observation related to this prediction is that the corresponding energies are accessible with existing or future data from HERA and the LHC.

\section{Photoproduction off nuclei}

We have also computed the photoproduction of $\rho^{0}$ mesons off nuclear targets for the coherent and the incoherent processes for three nuclei: $\mathrm{Xe}, \mathrm{Pb}, \mathrm{Au}$. Data are available for these three nuclei, or new measurements are expected in the near future. The results are shown in Table 1. One interesting result is that the coherent cross section, when computed using Eq. 2.4, develops an extra energy dependance due to the fluctuations of the subnucleon degrees of freedom. This is discussed in detail in [3].

\section{Conclusions and outlook}

We have presented results for the photoproduction of vector mesons off proton/nuclear targets for exclusive/coherent and dissociative/incoherent processes. We have shown that in particular the dissociative cross section shows a clear pattern of saturation dependance on the vector meson mass, which could be measured using existing HERA and LHC data or data to be recorded at the LHC in the near future. 

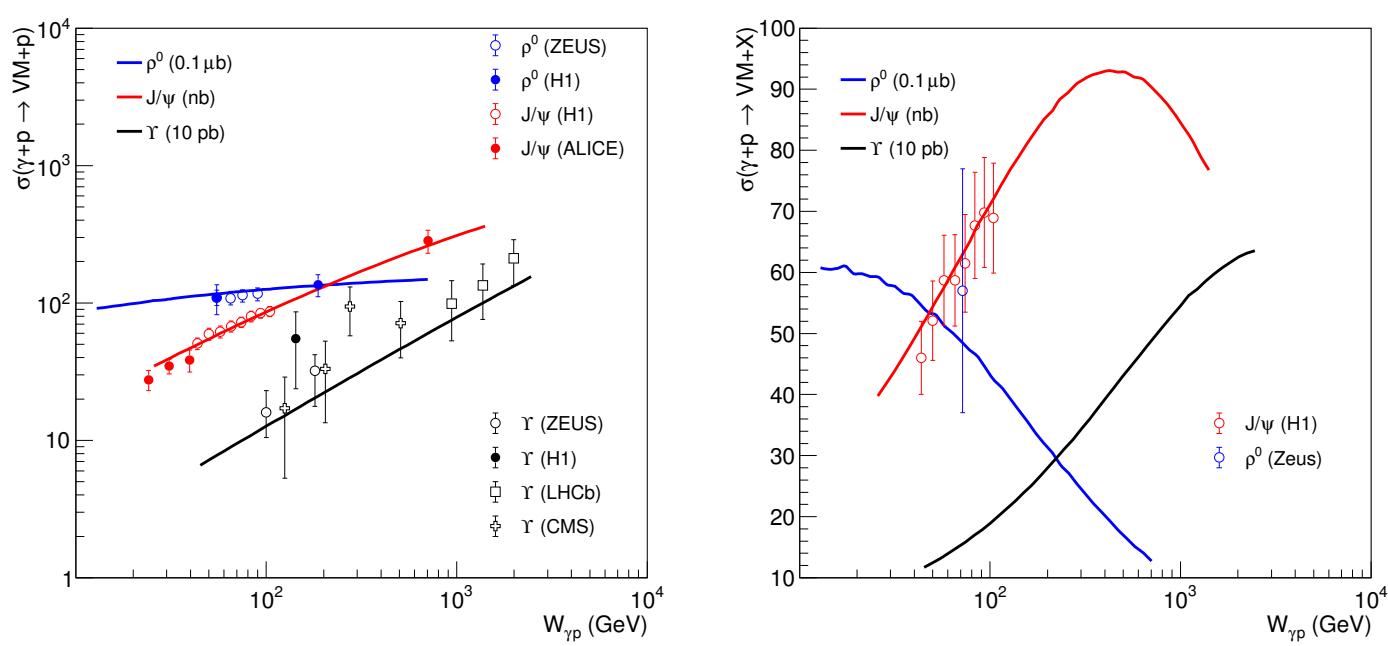

Figure 3: Exclusive (left) and dissociative (right) production of $\rho^{0}, \mathrm{~J} / \psi$ and $\Upsilon(1 S)$ vector mesons off protons as a function of $W_{\gamma \mathrm{p}}$ as computed in our model (solid lines) compared to data from HERA and the LHC. Taken from [3].

Table 1: Cross sections for the coherent and the incoherent photonuclear production of $\rho^{0}$ at mid-rapidity in ultra-peripheral $\mathrm{Au}-\mathrm{Au}, \mathrm{Pb}-\mathrm{Pb}$ and $\mathrm{Xe}-\mathrm{Xe}$ collisions at energies available at RHIC and the LHC. The cross sections are computed with the use of Eq. 2.4. Values taken from [3].

\begin{tabular}{cccc}
\hline \hline System & $\sqrt{s_{\mathrm{NN}}}(\mathrm{TeV})$ & Coherent $(\mathrm{mb})$ & Incoherent $(\mathrm{mb})$ \\
\hline $\mathrm{Au}-\mathrm{Au}$ & 0.20 & 102 & 4.4 \\
$\mathrm{~Pb}-\mathrm{Pb}$ & 2.76 & 480 & 27 \\
$\mathrm{~Pb}-\mathrm{Pb}$ & 5.02 & 579 & 31 \\
$\mathrm{Xe}-\mathrm{Xe}$ & 5.44 & 160 & 9.8 \\
\hline \hline
\end{tabular}

Acknowledgements This work was partially supported by grants 18-07880S of the Czech Science Foundation, LTC17038 of the INTER-EXCELLENCE program at the Ministry of Education, Youth and Sports of the Czech Republic, and by grants Conicyt PIA/ACT 1406 (Chile) and Conicyt PIA/Basal FB0821 (Chile). Access to computing and storage facilities of the National Grid Infrastructure MetaCentrum provided under the programme CESNET LM2015042 of the Czech Republic is greatly appreciated.

\section{References}

[1] J. Cepila, J. G. Contreras and J. D. Tapia Takaki, Energy dependence of dissociative J/ $\psi$ photoproduction as a signature of gluon saturation at the LHC, Phys. Lett. B766 (2017) 186-191, [1608.07559].

[2] J. Cepila, J. G. Contreras and M. Krelina, Coherent and incoherent $\mathrm{J} / \psi$ photonuclear production in an energy-dependent hot-spot model, Phys. Rev. C97 (2018) 024901, [1711.01855]. 
[3] J. Cepila, J. G. Contreras, M. Krelina and J. D. Tapia Takaki, Mass dependence of vector meson photoproduction off protons and nuclei within the energy-dependent hot-spot model, Nucl. Phys. B934 (2018) 330-340, [1804.05508].

[4] K. J. Golec-Biernat and M. Wusthoff, Saturation effects in deep inelastic scattering at low $Q^{* * 2}$ and its implications on diffraction, Phys. Rev. D59 (1998) 014017, [hep-ph/9807513].

[5] A. G. Shuvaev, K. J. Golec-Biernat, A. D. Martin and M. G. Ryskin, Off diagonal distributions fixed by diagonal partons at small $x$ and xi, Phys. Rev. D60 (1999) 014015, [hep-ph/9902410].

[6] H1 collaboration, S. Aid et al., Elastic photoproduction of rho0 mesons at HERA, Nucl. Phys. B463 (1996) 3-32, [hep-ex/9601004].

[7] ZEUS collaboration, J. Breitweg et al., Elastic and proton dissociative $\rho^{0}$ photoproduction at HERA, Eur. Phys. J. C2 (1998) 247-267, [hep-ex/9712020].

[8] H1 collaboration, C. Adloff et al., Elastic photoproduction of J / psi and Upsilon mesons at HERA, Phys. Lett. B483 (2000) 23-35, [hep-ex/ 0003020 ].

[9] ZEUS collaboration, S. Chekanov et al., Exclusive photoproduction of upsilon mesons at HERA, Phys. Lett. B680 (2009) 4-12, [0903 . 4205].

[10] H1 collaboration, C. Alexa et al., Elastic and Proton-Dissociative Photoproduction of J/psi Mesons at HERA, Eur. Phys. J. C73 (2013) 2466, [1304.5162].

[11] ALICE collaboration, B. B. Abelev et al., Exclusive J/ $\psi$ photoproduction off protons in ultra-peripheral p-Pb collisions at $\sqrt{s_{\mathrm{NN}}}=5.02$ TeV, Phys. Rev. Lett. 113 (2014) 232504, [1406.7819].

[12] LHCB collaboration, R. Aaij et al., Measurement of the exclusive $\Upsilon$ production cross-section in pp collisions at $\sqrt{s}=7 \mathrm{TeV}$ and $8 \mathrm{TeV}$, JHEP 09 (2015) 084, [1505.08139].

[13] CMS Collaboration collaboration, C. Collaboration, Measurement of exclusive $Y$ photoproduction in pPb collisions at $\sqrt{s_{\mathrm{NN}}}=5.02 \mathrm{TeV}$, Tech. Rep. CMS-PAS-FSQ-13-009, CERN, Geneva, 2016. 